\section{Retroviral protease-like sequence in the yeast transposon Ty 1}

RECENTLY, Mellor et al. ${ }^{1}$ determined the nucleotide sequence of the $5^{\prime}$-terminal portion of the yeast transposon Ty 1 and identified two open reading frames, ORF1 and ORF2, which are in the same relative positions as the gag and pol coding sequences in retroviruses. In addition, they showed that Tyl produces a fusion protein by a specific frameshifting process similar to that used by retroviruses. We have shown that retroviruses, cauliflower mosaic virus (CaMV), Drosophila copialike element 17.6 and Syrian hamster intracisternal A-type particle (IAP) contain sequences homologous to the gagspecific protease p15 of Rous sarcoma virus (RSV) in the same relative positions $s^{2,3}$. Furthermore, these p15-related sequences contain a highly conserved stretch of amino acids which shows a close similarity with sequences around the active-site amino acids Asp-Thr-Gly of the acid protease family, suggesting that they possess a similar activity to that of acid proteases ${ }^{2}$.

A computer-assisted search for homology has revealed the presence of the

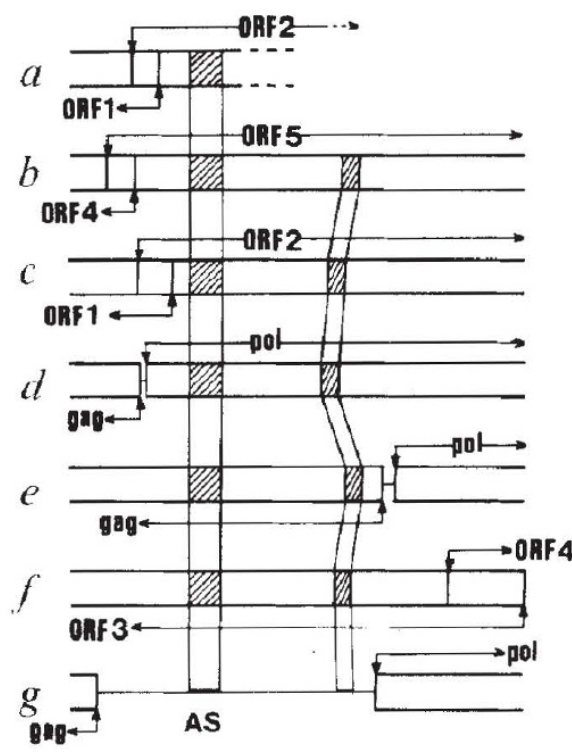

Fig. 1 Maps of homology between protease regions of reverse transcriptasecontaining viruses and transposons: $a$, Ty $1 ; b$, CaMV; $c, 17.6 ; d$, M-MuLV; $e$, RSV; $f$, IAP; g, HTLV-I (human adult T-cell leukaemia virus). Homologous segments are linked by slanted lines. No open reading frames are assigned between gag and pol in HTLV.I. Data were taken from ref. 2 for $b$-e and $g$, and from ref. 3 for $f$. $\mathrm{AS}$, the region showing a close sequence similarity with active-site amino acids of the acid protease family.

Table 1 Amino-acid sequence comparison between p15-related proteases and pepsin-related acid proteases

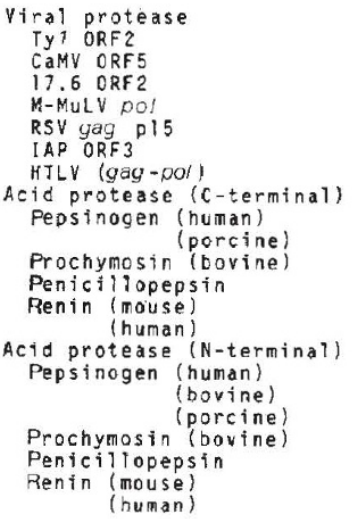

GHLLL-DSGASRTLIRS LHCFY-DTGASLCIA-S LKCLI - DTGSTVNMT -S VTFLV-DTGAQHSVL-T [TALL-DSGAOIT] I-S ITALI-DSGADITII - S IEALL - DTGADMTVL - $P$ COAIV-DTGTSLLTG - P CQAIV-DTGTSLLTG-P CQAIL-DTGTSKLVG-P FSGIA-DTGTTLLLL-B CEVVV-DTGSSF ISA-P CLALY-DTGASYISG-S DFTVYFOTGSSNLWVDFTVIFDTGSSNLWV-P DFTVIFDTGSSNLWV-P EFT VLFDTGSSDFWV-P TLNLNFOTGSADLWV - F TFKVMFDTGSANLWV-P TFKVVFDTGSSNVWV-P

As pepsin-related proteases consist of two topologically similar domains and two active-site aspartic acids, the amino-acid sequences around the two active sites were aligned between the $\mathrm{N}$ - and $\mathrm{C}$-terminal halves. Bold type indicates amino acids that are identical or chemically similar among 18 sequences $(90 \%)$ out of 20 at each position. Gaps(-) were inserted to increase sequence similarity. * Active sites of acid protease family. For sequence data, see refs 2 and 3.

p15-related sequence in the ORF2 of Ty 1 (Fig. 1, Table 1). Thus, the N-terminal portion of the ORF 2 product may have an activity similar to that of acid proteases. Such a widespread occurrence of the protease sequence suggests that it is vital for these viruses and transposons. Mellor et al. suggested that the fusion protein is processed proteolytically ${ }^{1}$; the ORF2coded protease may be involved in this processing. The relative positions of the conserved segments are almost the same among Ty $1, \mathrm{CaMV}, 17.6$ and Moloney murine leukaemia virus (M-MuLV) (Fig. $1)$, suggesting that the observed homologies are the result of divergence, not convergence. This also supports the notion that the ORF2 corresponds to pol in retroviruses $^{1}$, and thus the reverse transcriptase coding sequence, which is highly homologous among reverse transcriptasecontaining viruses and transposons ${ }^{2-5}$, probably lies in a region immediately downstream from the protease region.

\section{HIROYUKI TOH* MASAO ONO $\dagger$ KAORU SAIGO $\%$ TAKASHI MIYATA*}

* Department of Biology,

Faculty of Science,

Kyushu University, and

¥Department of Biochemistry,

Kyushu University School of Medicine,

Fukuoka 812, Japan
† Department of Molecular Biology, School of Medicine, Kitasato University, Sagamihara-shi, Kanagawa 228, Japan

1. Mellor, J et al Nature 313, 243-246 (1985).

2. Toh, $\mathrm{H}$. et al EMBO J. (in the press).

3. Ono, M., Toh, H., Miyata, T. \& Awaya, T. J. Virol. isub. mitted).

4. Toh, H., Hayashida, H. \& Miyata, T. Nature 305, 827-829 (1983)

5. Saigo, K. et al. Nature $312,659-661$ (1984)

MELLOR ET AL. REPLY-WE thank Toh et al. for their interest in our paper ${ }^{1}$ and for pointing out the homology with acid proteases at the beginning of ORF2 in Ty $1-15$. We would like to add that in pulse-chase experiments the protein, p1(Tyl-15), encoded by ORF1 in Ty $1-15$ seems to be processed, presumably by proteolytic cleavage. Furthermore, we have recently identified a candidate for the 'natural' product of ORF1/ORF2 fusion. This protein, p3(Ty1-15), has a relative molecular mass of 190,000 and can be seen only in short pulse-labelling experiments. Like p1(Ty1-15), therefore, it also appears to be proteolytically cleaved. We have observed four possible cleavage products and their relationships with p1(Ty1-15) and $\mathrm{p} 3(\mathrm{Ty} 1-15)$ are presently being estab. lished. The 'processing' of $\mathrm{pl}(\mathrm{Ty} 1-15)$ is dependent on the expression of ORF2, a result that is consistent with the processing protease being encoded within this region and therefore in agreement with the 